\title{
«Neumología ocupacional y ambiental». Temas necesarios en la formación del especialista en Medicina Respiratoria
}

\author{
Ireri Thirión-Romero, Laura Gochicoa-Rangel, Luis Torre-Bouscoulet $\bowtie$
}

Instituto Nacional de Enfermedades Respiratorias Ismael Cosío Villegas, Ciudad de México. Trabajo recibido: 08-VIII-2017; aceptado: 14-VIII-2017

En un año, un individuo cuya jornada laboral dura ocho horas por día, respira alrededor de 850 mil litros de aire que provienen de su ambiente de trabajo. Los gases, humos o partículas contenidas en esa inmensa cantidad de aire pueden afectar al sistema respiratorio de diversas formas y, eventualmente, generar síntomas respiratorios. ${ }^{1}$ Con excepción de las enfermedades por inhalación de polvos inorgánicos, la contribución del ambiente de trabajo en el desarrollo o exacerbaciones de enfermedades respiratorias está subestimada. En términos generales, se acepta que las exposiciones ocupacionales explican el $15 \%$ de los casos de asma y entre el 15 y $20 \%$ de los casos de enfermedad pulmonar obstructiva crónica (EPOC); este porcentaje alcanza el $40 \%$ en casos de EPOC en no fumadores. El 15\% de los casos de cáncer pulmonar en hombres y $5 \%$ en mujeres, se asocian a exposiciones ocupacionales. ${ }^{2}$

Un estudio epidemiológico llevado a cabo en Francia demostró que exposiciones ocupacionales de baja intensidad en lugares no industriales, también son un factor de riesgo para la salud respiratoria. ${ }^{3}$ En países en desarrollo estas cifras seguramente son mayores. Las minorías étnicas y el pobre estado socioeconómico son factores de riesgo para daños respiratorios originados por contaminantes del aire ambiental y por exposiciones ocupacionales. ${ }^{4}$ En el estudio PLATINO, $21.2 \%$ de los sujetos no fumadores con obstrucción fija tuvieron exposición laboral por más de cinco años a polvos o humos. ${ }^{5}$

La carga de enfermedad asociada a los contaminantes del aire en lugares de trabajo se ha incrementado progresivamente en los últimos años. Una causa de ello es el reconocimiento reciente de enfermedades pulmonares ocupacionales. En 2004, por ejemplo, se describieron casos de bronquiolitis obliterante rápidamente progresiva debida a exposición a saborizantes de palomitas de maíz (diacetil). ${ }^{6}$ El marcador funcional de esta enfermedad es obstrucción bronquial irreversible y pérdida acelerada de volumen espiratorio forzado en el primer segundo. Otras enfermedades pulmonares ocupacionales recién reconocidas incluyen a la enfermedad pulmonar por metales pesados, pulmón del pulidor de diamantes, pulmón asociado al síndrome del edificio enfermo y síndrome de Ardystil (neumonía organizada asociada a la industria textil). ${ }^{2}$ La exposición a arsénico también es causa de pérdida acelerada de función pulmonar. ${ }^{7}$

Además de la pobre calidad del aire asociada al lugar de trabajo, el cambio climático y la contaminación ambiental afectan la salud respiratoria. Después de la hipertensión arterial sistémica y el tabaquismo, la contaminación del aire es ahora el tercer factor de riesgo más importante para mortalidad a nivel global. ${ }^{4} \mathrm{El}$ calentamiento global por los gases con efecto invernadero (especialmente $\mathrm{CO}_{2}$ ), tiene un efecto directo sobre el sistema respiratorio. La mayor temperatura y humedad del aire genera incremento significativo de la resistencia de la vía aérea, lo que se ha postulado como uno de los mecanismos de exacerbación de asma y EPOC. ${ }^{8}$ El incremento en la temperatura aumenta la ventilación minuto y aumenta con ello la exposición a alérgenos.

El ozono es otro agresor del sistema respiratorio. El incremento en la concentración de ozono debido a la emisión de los automóviles induce un estado inflamatorio del epitelio bronquial..$^{9,10} \mathrm{La}$ elevada concentración de ozono en la superficie terrestre, especialmente en las grandes ciudades, afecta negativamente a pacientes con enfermedades respiratorias crónicas como asma o EPOC. Algunos estudios han demostrado que elevadas concentraciones de ozono también incrementan la mortalidad respiratoria; Jerrett $\mathrm{M}$ et al. demostraron que por cada incremento de $10 \mathrm{ppb}$ de ozono, se incrementa la 
mortalidad respiratoria en $3 \% .{ }^{11}$ El material particulado también incrementa las visitas a urgencias de pacientes con asma o EPOC y es un riesgo independiente para pérdida acelerada de la función respiratoria, neumonía y bronquitis aguda. ${ }^{9}$

Los niños son especialmente vulnerables a los efectos tóxicos de los contaminantes. La contaminación del aire no sólo incrementa las exacerbaciones de asma o empeora los síntomas en niños y adultos con enfermedades respiratorias crónicas. También se ha demostrado que la contaminación del aire limita el desarrollo pulmonar de los niños con consecuencias negativas en la vida adulta. ${ }^{9}$

Los miles de litros de aire contaminado que respiramos diariamente con el incremento consecuente de la morbilidad y mortalidad respiratorias, es un tema que no puede pasarse por alto en la formación del neumólogo moderno. En el momento actual y en términos generales, la sección de enfermedades pulmonares ocupacionales en la formación del neumólogo se reduce al capítulo de las neumoconiosis. Aunque importante, este grupo de enfermedades no revela de forma detallada la magnitud de los daños a la salud respiratoria asociada a las exposiciones laborales o ambientales. La formación de los especialistas en medicina respiratoria debería incluir un robusto programa de Neumología Ocupacional y Ambiental. El neumólogo debe saber investigar, de forma detallada, la historia de exposiciones ocupacionales y ambientales. Los cuestionarios disponibles para dicho fin tienen especial aplicación en epidemiología respiratoria; sin embargo, también son útiles para su uso individual. La estandarización de los cuestionarios aumenta su validez y consistencia, lo cual es importante no sólo para identificar la exposición; sino también, para cuantificarla. El residente de neumología es experto para clasificar pacientes con EPOC o cáncer pulmonar, pero no sabe investigar ni cuantificar las exposiciones laborales o ambientales a las que ha sido sometido el paciente. También se requiere que el neumólogo sea capaz de implementar programas de vigilancia respiratoria de acuerdo a la naturaleza nociva de los gases o partículas que existen en un determinado lugar de trabajo. La espirometría y la flujometría son indispensables en los programas de vigilancia respiratoria. La implementación de la prueba de difusión pulmonar de monóxido de carbono en los programas de vigilancia respiratoria incrementaría notablemente la sensibilidad diagnóstica.

El estudio de la participación del ambiente en la generación de enfermedad respiratoria debería ser incluido en los programas académicos, en especial ahora con el cambio climático y el calentamiento global. Es necesario que el neumólogo moderno conozca de química ambiental, de susceptibilidad genética a daños asociados a contaminantes, de calentamiento global por gases con efecto invernadero, epigenética ocupacional o ambiental, toxicología, estrés oxidante, ozono, material particulado, medidas de protección personal, prevención respiratoria en desastres naturales 0 accidentes industriales, contingencias ambientales, emergencias epidemiológicas por mala calidad del aire, etcétera. Además, debe entender las interacciones entre las condiciones ambientales y las enfermedades respiratorias preexistentes.

La incorporación de la Neumología Ocupacional y Ambiental en los programas académicos de formación de neumólogos, es una necesidad urgente que ampliaría el campo de acción del especialista. Esto le permitiría no sólo atender las consecuencias de las exposiciones respiratorias; sino también involucrarse en las estrategias de prevención de los daños a la salud asociados a los contaminantes del aire. Somos los neumólogos quienes debemos ser líderes en salvaguardar, a toda costa, el derecho universal a respirar aire limpio.

\section{REFERENCIAS}

1. Sigsgaard T, Nowak D, Annesi-Maesano I, et al.; ERS EOH group 6.2. ERS position paper: work-related respiratory diseases in the EU. Eur Respir J 2010;35(2):234-238. doi: 10.1183/09031936.00139409.

2. European Respiratory Society. European Lung White Book. Occupational lung diseases. Fecha de consulta: 07-VIII-2017. Accesible en: http://www.erswhitebook. org/chapters/occupational-lung-diseases/

3. Krzyzanowski M, Kauffmann F. The relation of respiratory symptoms and ventilatory function to moderate occupational exposure in a general population. Results from the French PAARC study of 16,000 adults. Int J Epidemiol 1988;17(2):397-406.

4. Carlsten C, Georas SN. Update in environmental and occupational lung diseases 2013. Am J Respir Crit Care Med 2014;189(9):1037-1043. doi: 10.1164/rccm.201401$0108 U P$.

5. Perez-Padilla R, Fernandez R, Lopez-Varela MV, et al. Airflow obstruction in never smokers in five Latin American cities: the PLATINO study. Arch Med Res 2012;43(2):159-165. doi: 10.1016/j. arcmed.2012.03.007.

6. Centers for Disease Control and Prevention (CDC). Fixed obstructive lung disease among workers in the flavor-manufacturing industry--California, 20042007. MMWR Morb Mortal Wkly Rep 2007;56(16):389393.

7. Parvez F, Chen Y, Yunus M, et al. Arsenic exposure and impaired lung function. Findings from a large population-based prospective cohort study. Am J Respir Crit Care Med 2013;188(7):813-819. doi: 10.1164/ rccm.201212-22820C. 
8. Hayes D, Collins PB, Khosravi M, et al. Bronchoconstriction triggered by breathing hot humid air in patients with asthma: role of cholinergic reflex. Am J Respir Crit Care Med 2012;185(11):1190-1196. doi: 10.1164/rccm.201201$00880 C$.

9. Gerardi DA, Kellerman RA. Climate change and respiratory health. J Occup Environ Med 2014;56 Suppl 10:S49-S54. doi: 10.1097/JOM.0000000000000292.

10. Scannell C, Chen L, Aris RM, et al. Greater ozoneinduced inflammatory responses in subjects with asthma. Am J Respir Crit Care Med 1996;154(1):2429.
11. Jerrett $\mathrm{M}$, Burnett RT, Pope $\mathrm{CA}$, et al. Long-term ozone exposure and mortality. N Engl J Med 2009;360(11):10851095.

\section{$\triangle$ Correspondencia:}

Dr. Luis Torre-Bouscoulet

Subdirección de Investigación Clínica, Instituto

Nacional de Enfermedades Respiratorias Ismael

Cosío Villegas.

Calzada de Tlalpan Núm. 4502, colonia Sección XVI,

14080, Del. Tlalpan, CDMX.

Correo electrónico: luistorreb@gmail.com

Los autores declaran no tener conflicto de intereses. 